\title{
Bielmaging
}

\section{Analysis of Multidimensional Biological Image Data}

\author{
K.W. Eliceiri, C. Rueden, W.A. Mohler ${ }^{1}$, \\ W.L. Hibbard, and J.G. White \\ University of Wisconsin-Madison, Madison, \\ WI, USA and ${ }^{1}$ University of Connecticut Health \\ Center, Farmington, CT, USA
}

Relatively recent strides in noninvasive medical imaging and in vital microscopy have yielded a new form of massive data type: the multi-channel, 3-D, time-course image recording. These multidimensional datasets document dynamic changes within the full volume of a specimen over time, often simultaneously monitoring several different parameters. As a result, visual data collected from a single living specimen can now go beyond the 2-D domain, engaging the viewer's full capacity to discern changes across space, time, and additional dimensions such as image spectra. A challenge rising out of these advances is to display these data so that the investigator can visualize and interactively explore the recording's full spatial, temporal. and spectral content, to better understand what cannot be seen directly through the microscope eyepiece. The challenges of multidimensional image analysis are not unique to the biologist or microscopist - space scientists and climatologists have been struggling with these issues for some time in their analysis of atmospheric data. Here we discuss computational approaches to this type of data, including the introduction of a new interdisciplinary effort to develop an effective framework for the analysis of multidimensional image data.

\section{WHY MULTIDIMENSIONAL?}

Examples of multidimensional image data include $(i)$ optically sectioned image recordings obtained using confocal (14), multiphoton $(12,19)$, or deconvolution (10) of the distinct signals produced by an organelle-specific chemical probe during the development of an embryo; (ii) MRI tomography of a live patient's tissues responding to a treatment or the development of a mouse embryo (9); and (iii) a reconstruction of serially sectioned fixed specimens representing sequential stages in the changing ultrastructure of a particular cell type (4). While the imaging modalities used to generate the data differ greatly, they all share the common attributes of being multidimensional and are usually represented by fairly large data archives. There have been successful attempts by several groups (including ours) to develop software tools for acquiring and doing basic analyses on 3-D time-lapse (4-D) datasets $(5,8,15-17)$. Unfortunately, there has been little attempt to develop a framework for the advanced analysis and manipulation of multidimensional data (1). This is due in part to the potential size of these datasets; for example, a multiphoton embryo recording consisting of 85 timepoints and 30 slices (Figure 1) would be roughly 1 GB in size on disk. Because of the speed and low cost of computer hardware, it is quite reasonable to acquire this magnitude of data, yet its subsequent analysis presents a serious computational challenge. With a specially designed analysis framework for multidimensional images, these massive data archives could be more readily explored to extract all possible information and detail present in each dimension (18).

\section{WHY THE NEED FOR SOPHISTICATED ANALYSIS TOOLS?}

In each type of multidimensional dataset, the archived data provide the potential for repeated retrospective analysis of the experiment. In fact, these data are so rich in information that without repeated reanalysis by researchers focusing on different systems and hypotheses, much of their value will be wasted-hence the need for visualization software that would allow an individual biologist within the community to be able to reanimate the live specimen on the computer, explore its anatomy and ontogeny from different viewpoints, watch significant events on any chosen time scale, and focus on specific aspects of its optical signature.

Continuing improvements to microscopy hardware are allowing biologists to increase both the quantity and complexity of data gathered during 4-D recordings of dynamic biological processes, resulting in two novel challenges to the goal of analyzing the data to extract meaningful conclusions.

\section{Increased Spatiotemporal Resolution in Time-Lapse Recording}

Improved speed and sensitivity of detectors has dramatically increased the total number of pixels that can be captured during a recording, both by shortening the delay between captures and by extending the survival of the specimen. Biological processes occur on a variety of size and time scales, all of which are often encompassed in the data from one of these recordings. Therefore, an ideal analysis tool would enable the researcher to browse interactively through datasets of greater than $10 \mathrm{~GB}$ or 50000 images, with the ability to change the display settings, resolution, and speed of animation to match the nature of the particular phenomenon being observed and measured.

\section{Increased Dimensionality within Each Pixel of an Image}

This expansion is occurring on several levels: increased dynamic range of simple detectors, construction of detectors that extract the spectrum and/or lifetime of emission from each pixel, the advent of second and third harmonic imaging, and a fast-growing collection of fluorochromes, physiological indicators, and reporter gene products. The result is that an image may no longer be entirely representable in a three-parameter (red-green-blue or hue-saturation-value) color model on the computer screen. Special interactive tools must be employed, for example, to display the spectral profiles of a given feature and correlate them with its position within the greater image (Figure 2). 


\section{COMMERCIAL PACKAGES FOR MULTIDIMENSIONAL DATA ANALYSIS}

Given the rise in popularity of multidimensional imaging in the biological research community, an obvious place to look for the solution for image analysis is the commercial imaging market. Biological journal advertising pages and scientific meeting product shows are currently awash with commercially available 3-D rendering packages. However, while many of these programs perform extremely well in rendering and re-slicing 3-D image volumes, there are several aspects of their development and distribution that prevent them from supporting the current and future realities of multidimensional imaging. Adaptation to different input file formats, use of robust rendering algorithms, and adoption of tested solutions from the graphics programming community are all inhibited by restrictive licensing agreements and the narrow circle of developers who can impact the maturation of closed-source proprietary software.

Thus, there is a need for an analysis software development effort intent on incorporating new features - such as the ones described here and demanded by the forefront in biological imaging research-that are beyond the current scope of market demand. Such a software package would not be intended simply to recapitulate or even combine features of the several commercially available $3-\mathrm{D}$ rendering packages, although, out of necessity, it would, to some extent. Instead, such an effort should be most intent on incorporating new features. Ideally, such a development effort would have a positive effect on the commercial imaging market, with popular features adopted by the mainstream commercial tools.

\section{OPEN-SOURCE SOLUTIONS TO ANALYSIS}

Over the past few years, we have been developing a suite of software applications for visualizing, annotating, and archiving 4-D movies (References 11, 13, and 17, and http:// www.loci.wisc.edu/cambio). It allows users to move through and annotate a recorded 4-D sequence backwards and forwards in time or up and down in focus with smooth animation (Figure 3). Although our 4-D system has become a routine tool for embryological and developmental studies, we have come to appreciate the need to develop a more comprehensive and powerful tool for the visualization and analysis of multidimensional data where the dimensions can include values other than time and space. To meet this need, our biological laboratory recently investigated the use of software developed to visualize weather patterns $(7,11)$ to view $4-\mathrm{D}$ datasets of developing embryos. The current embodiment of this system (VisAD) has extensive rendering, slicing, animation, and 3-D visualization capabilities and has demonstrated considerable promise as a visualization tool for the dynamics of developmental biology.

From our own experience with image analysis, we understood that our colleagues at large must analyze these data de novo from their own research perspectives for the scientific value of these data to approach its full potential. Thus, we decided to create a flexible, easily extensible visualization tool, VisBio. In the initial development of VisBio, we have chosen the VisAD programming toolset as both the basis of its construction and the model for its free, open-source distribution and community-based testing, refinement, and elaboration. One of the main goals of VisBio is to build on and improve VisAD's handling and display of multidimensional pixels.

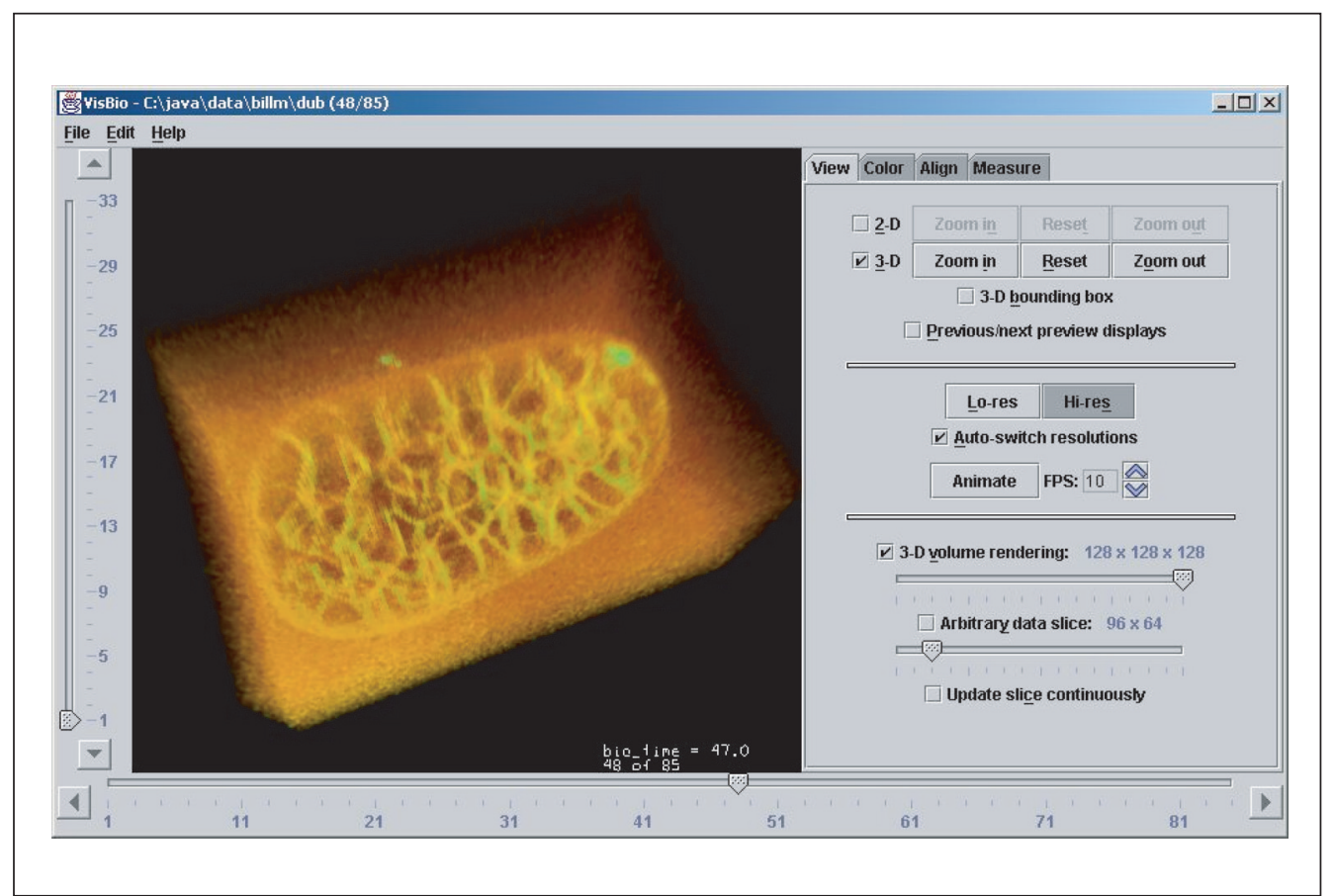

Figure 1. 3-D rendering of a live Caenorhabditis elegans embryo stained with a fluorescent membrane probe (FM4-64; Molecular Probes, Eugene, OR, USA). The interface allows free rotation of the rendered specimen, as well as dynamic data resolution scaling during animation through the 85-timepoint 1.2-GB recording. Imaging performed by William Mohler. 


\section{Bielmaging}

\section{BACKGROUND ON VisAD}

VisAD is a Java component library for interactive and collaborative visualization and analysis of numerical data (6). Programmers use this software library to create visualization applications for meteorologists, oceanographers, hydrologists, astronomers, economists, engineers, biologists, and others. These programmers download VisAD from servers at the University of Wisconsin-Madison, free of charge and including full source code and documentation (see http://www.ssec. wisc.edu/ billh/visad.html for details). This community of programmers communicates through the VisAD mailing list, answering each other's questions, reporting bugs, announcing software and documentation enhancements, and reviewing designs of planned enhancements. This model matches that of other, better-known open-source system communities such as Linux and Apache.

\section{PRELIMINARY DEVELOPMENT OF VisBio}

The VisBio software takes the form of an end-user application with a rich, yet focused feature set. The current implementation is a solid base for fluid user interaction in visualizing stacks of images in 3-D across multiple time steps, and it contains a number of important features.

Flexible color mapping (Figure 1). VisBio provides precise control over how colors are mapped. A dataset may have more than one color channel associated with each pixel. VisBio allows for complete control over each channel's color table including transparency.

Variable resolution (Figure 1). VisBio makes use of dual-resolution functionality, displaying data in lower resolution to improve animation speed, but "burning in" data at full resolution when more detail is needed for closer inspection. This approach not only improves rendering and animation speed but also cuts down on memory use because only the currently displayed time step must be maintained in memory at full resolution at any given time.

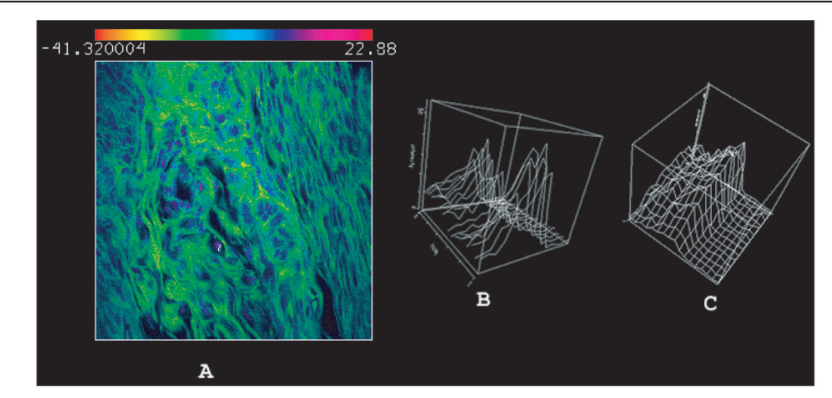

Figure 2. Seventeen-channel spectral image of a methyl green-stained section of a Cynomolgus monkey uterus imaged with multiphoton excitation. This mapping scheme was chosen to reveal nuclei as blue and surrounding tissue as green, although the actual spectral difference was subtle and not easy to see directly (A). Spectra of pixels along a short white line (B) reveal a peak at $600 \mathrm{~nm}$ and a shoulder on pixels over nuclei at $660 \mathrm{~nm}$ (upper left). Part $\mathrm{C}$ is a different visual projection of the same data represented in part B. Uterus section was prepared by Al Kutchera (Midwest Microtech, Cross Plains, WI)
Volume rendering (Figure 1). Support for rendering each image stack as a semi-transparent volume is implemented. Control over the level of transparency is provided, to locate the optimum visual setting for eliminating noise but preserving the important aspects of the data.

Arbitrary slicing. A plane can be placed at any orientation in the 3-D image stack, "slicing" it at any angle. Data along this arbitrary slice are interpolated and displayed at a user-defined resolution.

Measurement tools (Figure 4). A set of tools for performing measurements on the data is available. The distance between any two points in an image stack can be computed, and important events in the data can be flagged with markers.

\section{DEVELOPMENT MODEL}

VisBio has been built with the VisAD API and includes ImageJ (a widely adopted, public-domain Java image analysis system developed by Wayne Rasband at the NIH; http://rsb.info.nih.gov/ij/), and it therefore inherits a multitude of features from both tools. Work done to improve or enhance ImageJ or VisAD automatically benefits VisBio. More importantly, as additional or improved functionality in VisBio has been needed, we have implemented it in the core VisAD package, benefiting not only the biological community but also all users of VisAD in general.

\section{PLATFORM AVAILABILITY AND EXTENSIBILITY}

Since VisBio is built with Java2, it can run on any Java2enabled platform. The VisBio Web site (http://www.loci.wisc.

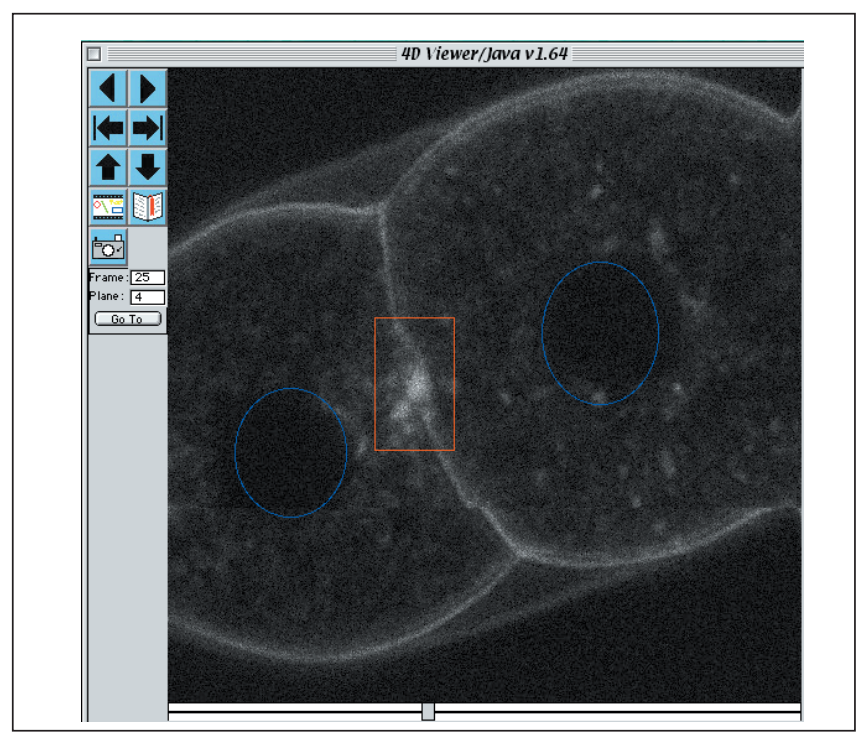

Figure 3. Screenshot from 4-D viewer showing an annotated multiphoton fluorescence excitation image of a recently divided $C$. elegans embryo stained with a membrane dye (FM-210; Molecular Probes). The blue circles show the nuclei, and the red box points out the membrane accumulation at the site of cleavage completion. Image provided by Dr. Jayne Squirrell (University of Wisconsin-Madison). 


\section{Bielmaging}

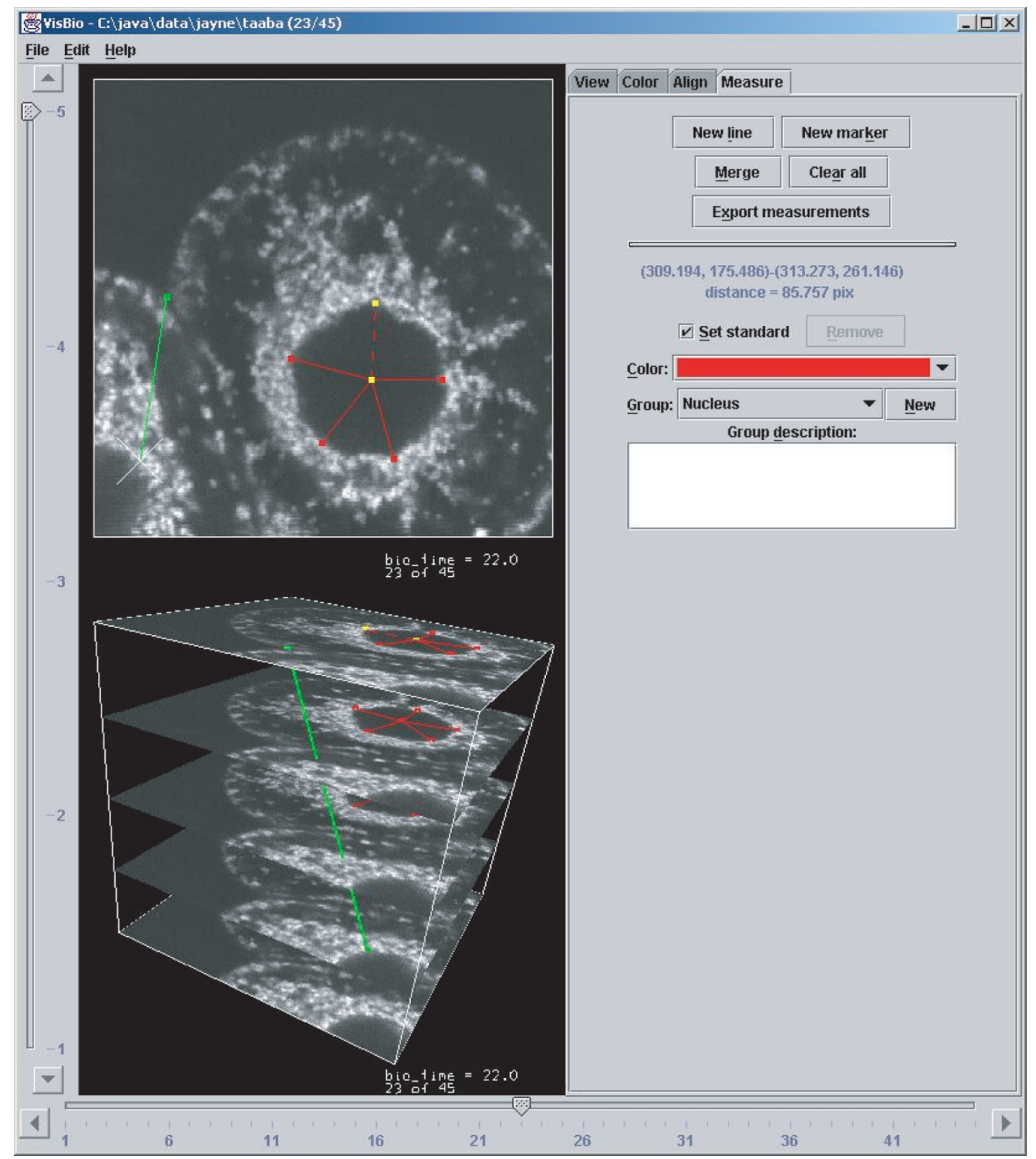

Figure 4. VisBio screenshot. 3-D and 4-D measurements quantifying movement of mitochondria in different regions of a two-photon dataset of a two-cell hamster embryo labeled with a mitochondria-specific dye, Mitotracker ${ }^{\circledR}-\mathrm{X}$-Rosamine (Molecular Probes). The images were collected as a $\mathrm{z}$ series of five optical sections, $0.5 \mu \mathrm{m}$ apart. Image provided by Dr. Jayne Squirrell.

edu/visbio) provides installers for Windows ${ }^{\circledR}$, Solaris (Sun Microsystems, Palo Alto, CA, USA), Linux, and Mac ${ }^{\circledR}$ OS X, to streamline the setup process. However, because VisBio utilizes Java3D for its 3-D displays, its full functionality is limited to those platforms that support Java3D. As of this writing, these platforms include Windows, Solaris, Linux, AIX, HPUX, and IRIX. VisBio can run when Java3D is not present, but many of its most useful features will be disabled because of the lack of 3-D display capabilities.

Because VisBio is open-source and object-oriented, Java programmers can build new components on top of VisBio to suit their own research needs. However, knowledge of Java is by no means a requirement for extending VisBio's capabilities. For scientists that do not know Java, VisBio supports an easy-to-use scripting language called Jython (a Java implementation of the widely used Python programming language). Flexible yet powerful, Jython is yet another component integrated with core VisAD, and Jython has a small learning curve compared to Java programming. Researchers accustomed to writing macros for specialized tasks with programs such as the popular NIH Image will see Jython's potential for cutting down on repetitive tasks as well as for providing new functionality.

\section{FUTURE DIRECTIONS}

We are developing computational strategies that would provide the capability of visualizing extremely large, multidimensional datasets within VisBio. The three main problems to be addressed are $(i)$ how to display multidimensional pixel data effectively, (ii) how to handle the processing of multi-gigabyte datasets with limited computer memory, and (iii) how to optimize computationally intense color operations so that it is possible to provide the animation that is so essential for the visualization of dynamic events. We plan to develop an architecture enabling the archival of a number of screen datasets that were obtained with different mapping functions so that a user can rapidly switch between different visual representations of the data. For example, separate screen datasets showing fluorescent lifetime and three-channel color could be set up, allowing a user to switch rapidly between the two different representations of the data. The proposed functionality will provide a unique and powerful tool for visualizing the dynamics of biological processes, using all the new dimensions of data that are becoming available from newly developed microscopies such as spectral (3), fluorescence lifetime, and harmonic imaging (2). 


\section{REFERENCES}

1.Andrews, P.D., I.S. Harper, and J.R. Swedlow. 2002. To 5D and beyond: quantitative fluorescence microscopy in the postgenomic era. Traffic 3:29-36.

2.Campagnola, P.J., A.C. Millard, M. Terasaki, P.E. Hoppe, C.J. Malone, and W.A. Mohler. 2002. Three-dimensional high-resolution secondharmonic generation imaging of endogenous structural proteins in biological tissues. Biophys. J. 82:493-508

3.Dickinson, M.E., G. Bearman, S. Tille, R. Lansford, and S.E. Fraser. 2002. Multi-spectral imaging and linear unmixing add a whole new dimension to laser scanning fluorescence microscopy. BioTechniques 31:1272-1278.

4.Duman, J.G., N.J. Pathak, M.S. Ladinsky, K.L. McDonald, and J.G.

Forte. 2002. Three-dimensional reconstruction of cytoplasmic networks in parietal cells. J. Cell Sci. 115:1251-1258.

5.Fire, A. 1994. A four-dimensional digital image archiving system for cell lineage tracing and retrospective embryology. Comput. Appl. Biosci. 10:443-447.

6.Hibbard, W., C. Rueden, S. Emmerson, T. Rink, D. Glowacki, D. Murray, T. Whittaker, D. Fulker, and J. Anderson. 2002. Java distributed objects for numerical visualization in VisAD. Commun. ACM 45:160-170.

7.Hibbard, W., S. Emmerson, C. Rueden, T. Rink, D. Glowacki, N. Rasmussen, D. Fulker, and J. Anderson. 1999. Collaborative visualization and computation in the earth sciences using VisAD. Preprints, Conf. Interactive Information and Processing Systems for Meteorology, Oceanography, and Hydrology. Dallas Amer. Meteor. Soc. 478-480.

8.Hird, S.N. and J.G. White. 1993. Cortical and cytoplasmic flow polarity in early embryonic cells of Caenorhabditis elegans. J. Cell Biol. $121: 1343-1355$.

9.Jacobs, R.E., E.T. Ahrens, T.J. Meade, and S.E. Fraser. 1999. Looking deeper into vertebrate development. Trends Cell Biol. 9:73-76.

10.McNally, J.G., T. Karpova, J. Cooper, and J.A. Conchello. 1999. Three dimensional imaging by deconvolution microscopy. Methods 19:373-385.

11.Mohler, W.A. 1999. Visual reality: using computer reconstruction and animation to magnify the microscopist's perception. Mol. Biol. Cell 10:3061-3065

12.Mohler, W.A. and J.G. White. 1998. Multiphoton laser scanning microscopy for four-dimensional analysis of C. elegans embryonic development. Optics Express. 3:325-331.

13.Mohler, W.A. and J.G. White. 1998. Stereo-4-D reconstruction and animation from living fluorescent specimens. BioTechniques 24:1006-1012.

14.Paddock, S.W. 1999. Confocal laser scanning microscopy. BioTechniques 27:992-1004.

15.Paddock, S.W. 2001. A brief history of time-lapse. BioTechniques 30:283-289.

16.Schnabel, R., H. Hutter, D. Moerman, and H. Schnabel. 1997. Assessing normal embryogenesis in Caenorhabditis elegans using a 4D microscope: variability of development and regional specification. Dev. Biol. 184:234-265

17.Thomas, C., P. DeVries, J. Hardin, and J. White. 1996. Four-dimensional imaging: computer visualization of $3 \mathrm{D}$ movements in living specimens. Science 273:603-607.

18.Uttecht, S. and K.R. Thulborn. 2002. Software for efficient visualization and analysis of multiple, large, multi-dimensional data sets from magnetic resonance imaging. Comput. Med. Imaging Graph. 26:73-89.

19.White, J.G., J.M. Squirrell, and K.W. Eliceiri. 2001. Applying multiphoton imaging to the study of membrane dynamics in living cells. Traffic 2:775-780.

Address correspondence to Kevin Eliceiri, LOCI, $1675 \mathrm{Ob}$ servatory Drive, University of Wisconsin-Madison, Madison, WI 53706,USA.e-mail:eliceiri@wisc.edu 